\title{
Shallow flows of generalised Newtonian fluids on an inclined plane
}

\author{
David Pritchard, Brian R. Duffy, and Stephen K. Wilson \\ Department of Mathematics and Statistics, University of Strathclyde, \\ 26 Richmond Street, Glasgow G1 1XH, Scotland, U. K.
}

June 21, 2014

\begin{abstract}
We derive a general evolution equation for a shallow layer of a generalised Newtonian fluid undergoing two-dimensional gravity-driven flow on an inclined plane. The flux term appearing in this equation is expressed in terms of an integral involving the prescribed constitutive relation and, crucially, does not require explicit knowledge of the velocity profile of the flow; this allows the equation to be formulated for any generalised Newtonian fluid. In particular, we develop general solutions for travelling waves on a mild slope and for kinematic waves on a moderately steep slope; these results provide simple and accessible models of, for example, the propagation of non-Newtonian mud and debris flows.
\end{abstract}

Keywords: lubrication flow; free-surface flow; thin film; non-Newtonian rheology.

\section{Introduction}

The dynamics of 'shallow' or 'slender' flows with a small aspect ratio have been a topic of scientific and mathematical investigation since the work of Reynolds [1]. A key feature of such flows is that the mathematical models that describe them can be asymptotically reduced to a simpler form, which captures the essential physics of the flow without requiring intensive computations. Such reduced models have been successfully employed in many contexts [2,3]. Further simplifications are possible because in shallow flows the reduced Reynolds number, the product of the aspect ratio and the standard Reynolds number, is typically small and so inertia may be neglected - the so-called 'lubrication approximation'.

A substantial body of work in shallow-flow theory was motivated by applications to largescale free-surface flows such as debris flows and lahars (see, e.g., [4]). In the simplest descriptions of these phenomena, a fixed volume of fluid is instantaneously released, and the aim of the model is to predict how far and how fast the resulting viscous gravity current can travel. Huppert [5, 6] considered two paradigm problems. In one problem [5], the fluid is released on a horizontal substrate, and after a period of initial adjustment the current spreads according to a self-similar solution. In the other problem [6], the fluid is released on a moderately steep slope, and propagates downslope as a kinematic wave [7, chapter 2]. Like other such waves [8], at large times the kinematic-wave solutions approach the 'centred-wave' solution that corresponds to an instantaneous point release of fluid. Huppert's analysis of the behaviour of such kinematic waves was later extended to account for the modification of the kinematic-wave solution near the front of the current by surface tension [9] or hydrostatic pressure gradients [10]. 
Although the theory of shallow flows was first developed for Newtonian fluids, many fluids of practical interest, ranging from lavas to colloidal suspensions such as muds, possess non-Newtonian rheological properties such as shear thinning and a yield stress [11]. There is therefore a substantial body of work in which lubrication theory has been extended to nonNewtonian fluids. For the problem of spreading on a horizontal substrate, notable contributions include those of Gorodtsov [12] and Pascal [13], who independently extended the self-similar solutions of [5] to power-law fluids; this work was later extended by Gratton et al. [14], who found a wider range of self-similar solutions. Another important contribution was made by Balmforth \& Craster [15], who demonstrated how lubrication theory could be formulated consistently for viscoplastic fluids; this work was built on to model the spreading of viscoplastic lava domes on a horizontal substrate [16] and viscoplastic coating flows [17].

Models of non-Newtonian flow on a slope have been independently developed by several researchers. One of the earliest contexts was glacier flow, since if basal slip and thermal effects are neglected, a glacier modelled using Glen's constitutive law obeys a power-law rheology with exponent $n=1 / 3$. Shallow-flow models, including kinematic-wave solutions, were constructed by Nye [18] and further developed by many researchers [e.g. 19, 20]; such models remain a topic of research [21]. Kinematic-wave models for a power-law fluid were presented inter alia by Berezhin et al. [22] and by Perazzo \& Gratton [23], while the explicit centred-wave solution has recently been presented and discussed by Ganguly et al. [24]. Huang \& Garcia [25] presented both the kinematic-wave solution and the front corrections for a viscoplastic Herschel-Bulkley fluid; the kinematic-wave solution has since been compared with experimental data by Ancey et al. [26]. More recently still, Kheyfets \& Kieweg [27] have presented a numerical study of unsteady shallow flows of an Ellis fluid on a slope.

Another class of solutions for flow on a slope is that in which the slope is mild. In this case, the governing lubrication equations admit travelling-wave solutions, which propagate downslope at a constant speed and can be regarded as representing the behaviour of an existing flow when the rate of supply of fluid is abruptly changed. Liu \& Mei [28] first developed such solutions for a Bingham fluid, while Perazzo \& Gratton [23, 29] presented a thorough analysis of the problem and the possible types of solution for a power-law fluid. More recently, Chambon et al. [30] have presented Herschel-Bulkley solutions and compared them with experiments that employed a kaolin slurry flowing on an inclined conveyor belt.

Extensions of the lubrication equations to inertial, but still laminar, flows have been presented for power-law, Bingham and Herschel-Bulkley fluids [31, 32, 33]. More recent developments in the study of non-Newtonian flows on slopes have largely focused on viscoplastic fluids, with a particular interest in the final deposit shape generated by slumping from various initial conditions $[34,35]$ and what can be deduced from it about the fluid rheology (the 'Bostwick consistometer' experiment [36]). A still more recent extension has been to gravity currents of thixotropic fluid on a slope [37].

So far, most shallow-flow models have been developed for relatively simple rheologies, for which velocity profiles in free-surface flow may be determined explicitly. However, such simple rheologies are not necessarily good descriptions of the behaviour of complex fluids such as mud. For more complicated and realistic rheological models, it is generally not possible to obtain velocity profiles explicitly: this has hindered the development of shallow-flow theory for complex fluids and may have encouraged a focus on mathematically tractable but unrealistic rheologies. In particular, the shear-thinning versions of the popular power-law and HerschelBulkley models both predict an infinite effective viscosity in the limit of low shear rate: this behaviour is physically incorrect (see, e.g., [38, chapter 1]) as well as being mathematically pathological, and may lead to physically misleading results; Myers [39] has argued that this issue, while well known, is often not properly acknowledged. 
In the present contribution, we address the need for a more general non-Newtonian shallowflow theory. We demonstrate in section 2 that because the stress distribution in shallow flows is known in advance, the governing equations for the unsteady shallow two-dimensional flow of any generalised Newtonian fluid can be written in terms of an integral of a function that is obtained directly from the constitutive law. Although the mathematical 'trick' that we employ is far from new - indeed, it was introduced by Weissenberg in his work on the capillary rheometer in 1928 (see, e.g., [40, §2.1]) - its full potential in this context does not seem to have been exploited. In the following sections, we specialise this general equation successively to two regimes in which the evolution equation simplifies substantially and particular forms of solution become available. Each regime has previously been investigated for Newtonian and power-law fluids but not for more general rheologies. First, in section 3, we consider the mild-slope regime in which the gradients of the inclined plane and of the free surface are comparable and travelling-wave solutions are available; then, in section 4, we consider the moderately-steep-slope regime in which the gradient of the inclined plane is dominant and a kinematic-wave description of the flow becomes appropriate. Having formulated these general problems, we illustrate them by presenting solutions in each case for the propagation of waves downslope, using the particular choice of a Carreau rheology to explore the effects of shear-thinning behaviour, and of Bingham and Casson rheologies where appropriate to explore the effects of yield-stress behaviour.

\section{Model description}

\subsection{Generalised Newtonian fluids}

The mass-conservation and momentum-balance equations for an incompressible fluid of density $\rho$ are

$$
\nabla \cdot \mathbf{u}=0
$$

and

$$
\rho\left(\frac{\partial \mathbf{u}}{\partial t}+\mathbf{u} \cdot \nabla \mathbf{u}\right)=-\nabla p+\rho \mathbf{g}+\nabla \cdot \boldsymbol{\sigma}
$$

where $\mathbf{u}, p$ and $\boldsymbol{\sigma}$ are the velocity, pressure and extra-stress tensor of the fluid, $\mathbf{g}$ denotes acceleration due to gravity, and $t$ denotes time.

The constitutive equation for a generalised Newtonian fluid [41, $\S 8.4]$ is of the form

$$
\boldsymbol{\sigma}=2 \mu(q) \mathbf{e}
$$

where $\mathbf{e}$ is the rate-of-strain tensor, given by

$$
\mathbf{e}=\frac{1}{2}\left(\nabla \mathbf{u}+(\nabla \mathbf{u})^{\mathrm{T}}\right)
$$

$q$ is the local shear rate, given by $q=\left(2 \operatorname{tr}\left(\mathbf{e}^{2}\right)\right)^{1 / 2}$, and $\mu=\mu(q)$ is a prescribed shear-rate-

dependent viscosity function. A measure of local extra stress is given by $\tau=\left(\frac{1}{2} \operatorname{tr}\left(\boldsymbol{\sigma}^{2}\right)\right)^{1 / 2}=$ $\mu(q) q$.

\subsection{Unsteady shallow flow down an inclined plane}

We consider the unsteady two-dimensional gravity-driven flow of a shallow layer of a generalised Newtonian fluid on a planar substrate inclined at an angle $\alpha \geq 0$ to the horizontal, as 


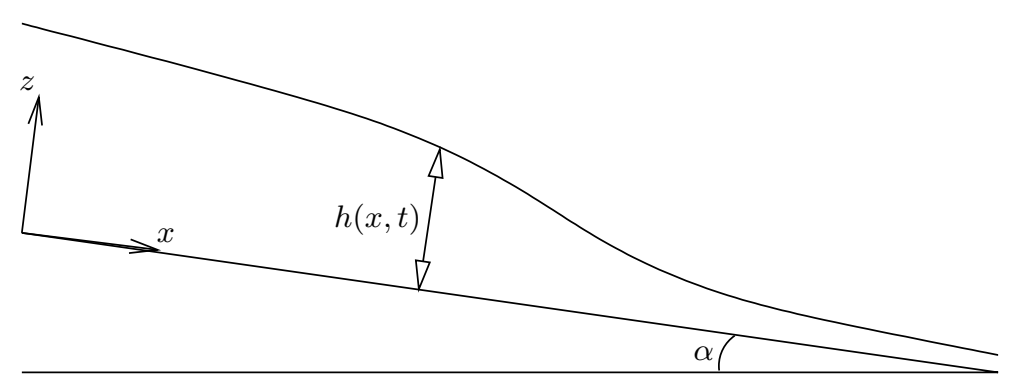

Figure 1: Schematic of a shallow two-dimensional flow on an inclined plane.

illustrated in figure 1. In terms of Cartesian coordinates $O x y z$ with the $x$ axis down the line of greatest slope, the $y$ axis horizontal and the $z$ axis normal to the substrate $z=0$, we denote the free surface of the fluid by $z=h(x, t)$. With a velocity of the form

$$
\mathbf{u}=u(x, z, t) \mathbf{i}+w(x, z, t) \mathbf{k}
$$

we have $q=|\partial u / \partial z|$ to the usual accuracy of shallow-layer theory (see, e.g., [3]), and equations (1) and (2) reduce in the lubrication approximation to

$$
\frac{\partial u}{\partial x}+\frac{\partial w}{\partial z}=0, \quad 0=-\frac{\partial p}{\partial x}+\rho g \sin \alpha+\frac{\partial}{\partial z}\left(\mu \frac{\partial u}{\partial z}\right), \quad 0=-\frac{\partial p}{\partial y}, \quad 0=-\frac{\partial p}{\partial z}-\rho g \cos \alpha,
$$

where $g=|\mathbf{g}|$ and $\mu=\mu(q)$. The equations (6) are to be integrated subject to the no-slip and no-penetration boundary conditions at the substrate,

$$
u=w=0 \text { on } \quad z=0,
$$

the kinematic condition at the free surface,

$$
\frac{\partial h}{\partial t}+u \frac{\partial h}{\partial x}=w \quad \text { on } \quad z=h
$$

and the tangential and normal stress conditions at the free surface,

$$
\frac{\partial u}{\partial z}=0 \quad \text { and } \quad p=p_{\mathrm{a}}-\gamma \frac{\partial^{2} h}{\partial x^{2}} \quad \text { on } \quad z=h,
$$

where $\gamma$ denotes the (constant) coefficient of surface tension and $p_{\mathrm{a}}$ denotes atmospheric pressure.

Integrating the continuity equation (1) over the depth of the fluid, subject to the boundary conditions (7) and (8), yields

$$
\frac{\partial h}{\partial t}+\frac{\partial Q}{\partial x}=0
$$

where $Q$ denotes the downslope volume flux of fluid in the $x$-direction (per unit width in the $y$ direction), given by

$$
Q=\int_{0}^{h} u \mathrm{~d} z=[(z-h) u]_{0}^{h}-\int_{0}^{h}(z-h) \frac{\partial u}{\partial z} \mathrm{~d} z=\int_{0}^{h}(h-z) \frac{\partial u}{\partial z} \mathrm{~d} z .
$$

Integrating equation (6c) in $z$ subject to the boundary condition (9b) yields

$$
p=p_{\mathrm{a}}+\rho g \cos \alpha(h-z)-\gamma \frac{\partial^{2} h}{\partial x^{2}},
$$


and then from $(6 b)$ we have

$$
\frac{\partial}{\partial z}\left(\mu(q) \frac{\partial u}{\partial z}\right)=-G
$$

where $G=G(x, t)$ is given by

$$
G=-\frac{\partial}{\partial x}\left(\rho g \cos \alpha h-\gamma \frac{\partial^{2} h}{\partial x^{2}}\right)+\rho g \sin \alpha .
$$

The importance of surface tension may be gauged in the usual way by defining a Bond number (Eötvös number) $B=\rho g \cos \alpha L^{2} / \gamma$, where $L$ is the horizontal lengthscale of the flow. Surface tension is then negligible provided that $B \gg 1$ and $B(L / H) \tan \alpha \gg 1$, where $H$ is the typical depth of the flow. These conditions are readily satisfied for fluids with comparable properties to water if the flow is of the order of centimetres deep and the shallow-flow condition is satisfied. Consequently, from this point onward unless otherwise stated we will take $\gamma=0$ and thus neglect any effects of surface tension. We also confine the discussion to slopes for which $0 \leq \alpha<\pi / 2$, so the substrate is not overhanging.

Integrating (13) once and using (9) we have

$$
\mu(q) \frac{\partial u}{\partial z}=G(h-z) .
$$

We now restrict our attention to free-surface flows in which $\partial u / \partial z \geq 0$ everywhere, so that $q=\partial u / \partial z$. Then from (15)

$$
\mu(q) q=G(h-z)
$$

so it follows that $G \mathrm{~d} z=-\{\mathrm{d}[\mu(q) q] / \mathrm{d} q\} \mathrm{d} q$. From (11), the flux $Q$ may therefore be written in the form

$$
Q=\frac{F\left(q_{0}\right)}{G^{2}},
$$

where $F\left(q_{0}\right)$ is a function that depends only on the rheology of the fluid, and is defined by

$$
F\left(q_{0}\right)=\int_{0}^{q_{0}} \mu(q) q^{2} \frac{\mathrm{d}[\mu(q) q]}{\mathrm{d} q} \mathrm{~d} q
$$

and where $q_{0}=q_{0}(x, t)=\left.q\right|_{z=0}$ is the shear rate at the substrate, satisfying

$$
\mu\left(q_{0}\right) q_{0}=G h .
$$

We note that the change of variables from $z$ to $q$ is possible only for rheological models in which the shear stress is a monotonic function of the shear rate. This is the case for all commonly employed generalised Newtonian rheologies, but not for some more exotic rheologies obtained, for example, as the generalised-Newtonian limit of a thixotropic model [37]. We also note that equation (17), multiplied by $G$, can be interpreted as an energetic balance between the rate of work done by pressure forces acting on a vertical section and the rate of dissipation of energy by the basal shear stress.

Substituting (17) into the kinematic condition (10) we obtain the partial differential equation governing the evolution of the free surface height $h(x, t)$ :

$$
\frac{\partial h}{\partial t}+\frac{\partial}{\partial x}\left(\frac{F\left(q_{0}\right)}{G^{2}}\right)=0
$$

in which $q_{0}$ is determined implicitly in terms of $G h$ by the algebraic equation (19), and $G$ is given by (14). The advantage of this over the original formulation which integrates (16) in $z$ is 
that only one implicit equation and one integration must be carried out at every point $(x, t)$; by contrast, the original formulation requires equation (16) to be inverted at enough $z$-points to obtain $Q$ via the last integral of (11). For rheologies in which (16) cannot be inverted explicitly, this is a significant saving in computational effort.

If the constitutive equation of the fluid is prescribed in the form $q=\phi(\tau)$ (rather than in the form $\mu=\mu(q)$ or the equivalent form $\tau=\tau(q)=\mu(q) q)$ then (18), (19) and (20) may be written more simply as

$$
\hat{F}\left(\tau_{0}\right)=\int_{0}^{\tau_{0}} \phi(\tau) \tau \mathrm{d} \tau, \quad \tau_{0}=G h, \quad \frac{\partial h}{\partial t}+\frac{\partial}{\partial x}\left(\frac{\hat{F}(G h)}{G^{2}}\right)=0,
$$

respectively. We will see in section 4.2.2 that this formulation is particularly useful when describing the flow of a fluid with a yield stress.

The evolution equations (20) and (21) constitute the most general results presented in this study. To demonstrate the capabilities of this approach, in the following sections we specialise to particular regimes of the slope of the incline, first a mild slope and then a moderately steep slope, and seek solutions for specific rheologies in each regime. Before doing so, we note briefly that we can recover the standard results for a power-law fluid from equation (20). If we set $\mu(q)=\mu_{n} q^{n-1}$, where $\mu_{n}$ and $n$ are positive material parameters, equation (19) gives $q_{0}=\left(G h / \mu_{n}\right)^{1 / n}$, and then (20) leads to

$$
\frac{\partial h}{\partial t}+\frac{n}{(2 n+1) \mu_{n}^{1 / n}} \frac{\partial}{\partial x}\left(G^{1 / n} h^{(2 n+1) / n}\right)=0
$$

(cf. [14]). When $n=1$ this reduces in turn to the well-known result for a Newtonian fluid (see, e.g., [5]).

\section{Flow on a mild slope: travelling waves}

We first consider the regime in which the slope is mild, $0<\alpha \ll 1$. In this regime we may consistently seek solutions of the lubrication equations for which $|\partial h / \partial x|=O(\tan \alpha)$ and so the hydrostatic pressure and alongslope gravitational contributions to $G$ are comparable in magnitude. Then from (14) and (19) we have

$$
G=\rho g \sin \alpha-\rho g \cos \alpha \frac{\partial h}{\partial x} \quad \text { and } \quad \mu\left(q_{0}\right) q_{0}=G h .
$$

(Note that in this regime $\sin \alpha \sim \alpha$ and $\cos \alpha \sim 1$; as both factors will be removed by scaling we do not need to employ this approximation.)

In this regime, the problem that has received most attention $[23,28,29,30]$ is that of travelling waves, which may be expected to represent the response of a uniform downslope flow to an abrupt increase in the supply of fluid from upstream. Generalising the results obtained for power-law fluids by Perazzo \& Gratton [29], we will seek travelling-wave solutions of the form $h(x, t)=H(\eta)$, where $\eta=x-c t$ for some constant wave velocity $c$. Equation (20) then becomes

$$
-c \frac{\mathrm{d} H}{\mathrm{~d} \eta}+\frac{\mathrm{d}}{\mathrm{d} \eta}\left(\frac{F\left(q_{0}\right)}{G^{2}}\right)=0,
$$

where $G=G\left(H^{\prime}\right)$ and $q_{0}=q_{0}\left(H, H^{\prime}\right)$ are given by

$$
G=\rho g \sin \alpha-\rho g \cos \alpha H^{\prime} \quad \text { and } \quad \mu\left(q_{0}\right) q_{0}=\left(\rho g \sin \alpha-\rho g \cos \alpha H^{\prime}\right) H,
$$


and where $F\left(q_{0}\right)$ is defined by (18).

Equation (24) can be integrated immediately to give

$$
L\left(H, H^{\prime}\right) \equiv-c H+\frac{F\left(q_{0}\left(H, H^{\prime}\right)\right)}{\left[G\left(H^{\prime}\right)\right]^{2}}=L_{0}
$$

for some constant $L_{0}$ which measures the downslope flux of fluid seen in the frame of the travelling wave. The constants $c$ and $L_{0}$ between them define a particular travelling wave solution, which can be thought of as a trajectory defined by (26) in the $\left(H, H^{\prime}\right)$ phase plane. Once such a solution has been defined, equation (26) can in principle be inverted to provide $H^{\prime}(H)$ along this trajectory, and the solution can then be written in implicit form as

$$
\eta=\int \mathrm{d} \eta=\int \frac{\mathrm{d} H}{H^{\prime}(H)} .
$$

\subsection{Travelling-wave solutions for a Carreau fluid}

To illustrate the approach, we will present solutions for the Carreau constitutive model [38, $\S 1.5]$, which has not previously been considered in this context. The constitutive equation for a Carreau fluid is (3) with $\mu(q)$ given by

$$
\mu=\mu_{\infty}+\frac{\mu_{0}-\mu_{\infty}}{\left(1+\lambda^{2} q^{2}\right)^{(1-n) / 2}},
$$

where $\lambda, n, \mu_{0}$ and $\mu_{\infty}$ are (positive) material parameters, $\lambda$ having the dimensions of time, $\mu_{0}$ and $\mu_{\infty}$ being the viscosities at low and high shear rates, respectively, and $n$ satisfying $n<1$. Commonly $\mu_{0}$ is taken to be larger (sometimes much larger) than $\mu_{\infty}$, corresponding to a shearthinning fluid. (The case $\mu_{0}<\mu_{\infty}$ corresponds to a shear-thickening fluid; since such fluids are rare in practice we do not consider them here.) The reciprocal of the parameter $\lambda$ is the characteristic shear rate associated with the transition from low- to high-shear-rate behaviour. The parameter $n$ controls how rapidly this transition occurs; its effect on the travelling-wave profiles is negligible and so for brevity we will not explore it here.

We assume that it is possible to identify a characteristic depth $H_{0}$ associated with the solution (typically the limiting depth of the fluid far up- or downstream), and we define dimensionless quantities, denoted by an asterisk, via

$$
H(\eta)=H_{0} H^{*}\left(\eta^{*}\right), \quad \eta=\frac{H_{0}}{\tan \alpha} \eta^{*}, \quad q=\frac{\rho g \sin \alpha H_{0}}{\mu_{0}} q^{*}, \quad c=\frac{\rho g \sin \alpha H_{0}^{2}}{\mu_{0}} c^{*} .
$$

The dimensionless viscosity $\mu^{*}\left(q^{*}\right)$ corresponding to (28) is given by

$$
\mu\left(q^{*}\right)=\mu_{\infty}^{*}+\frac{1-\mu_{\infty}^{*}}{\left[1+\left(\lambda^{*} q^{*}\right)^{2}\right]^{(1-n) / 2}}, \quad \text { where } \quad \mu_{\infty}^{*}=\frac{\mu_{\infty}}{\mu_{0}}, \quad \lambda^{*}=\frac{\rho g \sin \alpha H_{0} \lambda}{\mu_{0}} .
$$

The parameter $\mu_{\infty}^{*}$ determines whether the fluid is shear-thinning $\left(\mu_{\infty}^{*}<1\right)$ or shear-thickening $\left(\mu_{\infty}^{*}>1\right)$, while the parameter $\lambda^{*}$ determines whether a layer of fluid of constant depth $H_{0}$, flowing downslope under its own weight, is in a high-shear-rate regime $\left(\lambda^{*} \ll 1\right)$ or a lowshear-rate regime $\left(\lambda^{*} \gg 1\right)$.

The dimensionless forms of equations (25) are

$$
G^{*}=1-\frac{\mathrm{d} H^{*}}{\mathrm{~d} \eta^{*}} \quad \text { and } \quad \mu^{*}\left(q_{0}^{*}\right) q_{0}^{*}=G^{*} H^{*}
$$


and the dimensionless form of equation (18) is

$$
F^{*}\left(q_{0}^{*}\right)=\int_{0}^{q_{0}^{*}} \mu^{*}\left(q^{*}\right) q^{* 2} \frac{\mathrm{d}}{\mathrm{d} q^{*}}\left[\mu^{*}\left(q^{*}\right) q^{*}\right] \mathrm{d} q^{*} .
$$

Finally, the integrated governing equation (26) becomes

$$
L^{*}\left(H^{*}, H^{* \prime}\right) \equiv-c^{*} H^{*}+\frac{F^{*}\left(q_{0}^{*}\left(H^{*}, H^{* \prime}\right)\right)}{\left[G^{*}\left(H^{* \prime}\right)\right]^{2}}=L_{0}^{*} .
$$

A variety of travelling wave solutions are available [29]. We will pay particular attention to the case where the fluid asymptotically approaches a uniform depth of $h_{-\infty}$ upstream and a uniform depth of $h_{\infty}<h_{-\infty}$ downstream. This provides, for example, a simple model of a surge in a slow mud flow [28]. Taking $H_{0}=h_{-\infty}$ in (29), we obtain the dimensionless boundary conditions

$$
H^{*} \rightarrow 1 \quad \text { as } \quad \eta^{*} \rightarrow-\infty \quad \text { and } \quad H^{*} \rightarrow H_{\infty}^{*} \quad \text { as } \quad \eta^{*} \rightarrow \infty,
$$

where $H_{\infty}^{*}=h_{\infty} / h_{-\infty}$ is the dimensionless downstream depth. Without loss of generality we will set $\eta^{*}=0$ where $H^{*}=\left(1+H_{\infty}^{*}\right) / 2$ to allow a simple comparison between profiles for different parameter values.

We may determine the values of $c^{*}$ and $L_{0}^{*}$ immediately by solving the coupled equations

$$
-c^{*}+\frac{F^{*}\left(q_{0}^{*}(1,0)\right)}{\left[G^{*}(0)\right]^{2}}=L_{0}^{*} \quad \text { and } \quad-c^{*} H_{\infty}^{*}+\frac{F^{*}\left(q_{0}^{*}\left(H_{\infty}^{*}, 0\right)\right)}{\left[G^{*}(0)\right]^{2}}=L_{0}^{*} ;
$$

noting that $G^{*}(0)=1$, we obtain

$$
c^{*}=\frac{F^{*}\left(q_{0}^{*}(1,0)\right)-F^{*}\left(q_{0}^{*}\left(H_{\infty}^{*}, 0\right)\right)}{1-H_{\infty}^{*}}
$$

and

$$
L_{0}^{*}=\frac{F^{*}\left(q_{0}^{*}\left(H_{\infty}^{*}, 0\right)\right)-F^{*}\left(q_{0}^{*}(1,0)\right) H_{\infty}^{*}}{1-H_{\infty}^{*}} .
$$

For a given value of $H^{*}$ between $H_{\infty}^{*}$ and 1, the corresponding value of $H^{* \prime}$ may be obtained by solving (33) numerically; $\eta^{*}$ may then be obtained by quadrature from equation (27) and plotted against $H^{*}$. (The apparently more natural process of parameterising both $H^{*}$ and $\eta^{*}$ by $q_{0}^{*}$ is complicated by the non-monotonic variation of $H^{*}$ with $q_{0}^{*}$ which we will remark upon below, and so we do not pursue this alternative representation of the solution here.)

Figure 2 illustrates some typical solutions, spanning three decades of variation in $\lambda^{*}$. All the profiles have the same general shape (figures 2(a) and (b)): a blunt 'nose', smoothly connected by a small 'lip' to the fluid of depth $H_{\infty}^{*}$ ahead of the wave. The shape of the 'nose' is reminiscent of the shape of the front of a viscous gravity current over a dry bed [5], but differs from it by joining smoothly to the downstream region rather than having a singular gradient at the front. For smaller values of $H_{\infty}^{*}$, the nose is more strongly pronounced and the lip less obvious. Both the basal shear rate $q_{0}^{*}$ and the basal shear stress $\tau^{*}=\mu^{*}\left(q_{0}^{*}\right) q_{0}^{*}$ peak within the steeply-sloping nose (figures 2(c) and (d)), where the hydrostatic pressure gradient term $H^{* \prime}\left(\eta^{*}\right)$ is large. This peak is again most strongly pronounced when $H_{\infty}^{*}$ is small, as in figure 2 ; in the limit as $H_{\infty}^{*} \rightarrow 0$, the shear rate and bed shear stress in fact become infinite at the front. 
(a)

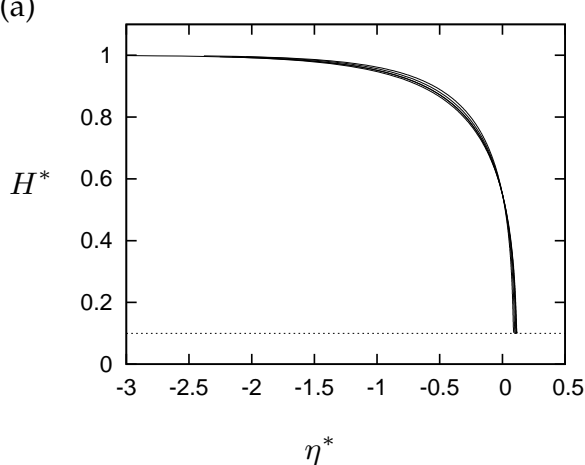

(c)

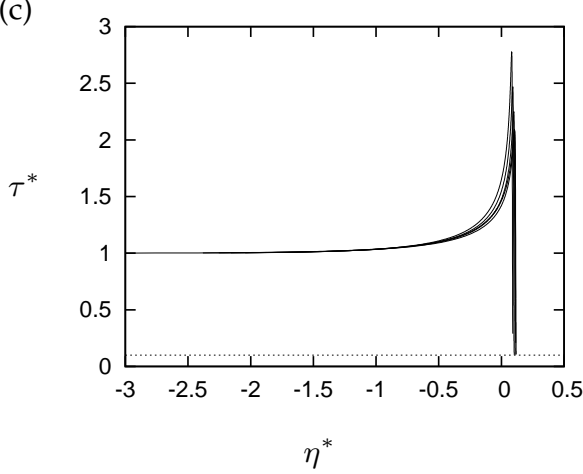

(b)

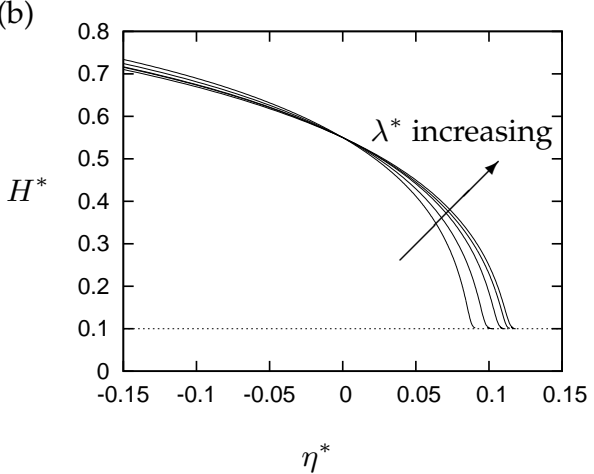

(d)

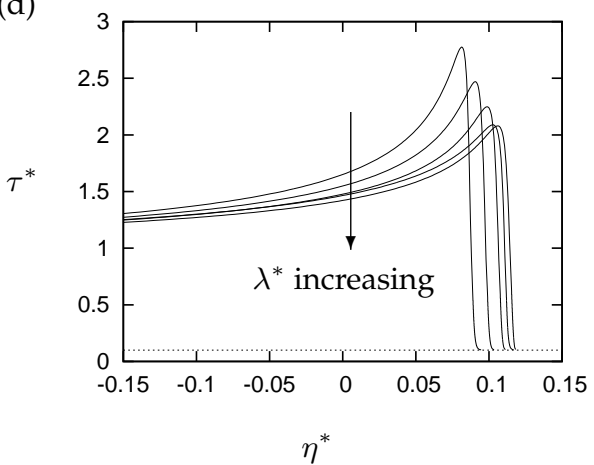

Figure 2: Travelling-wave solutions for a Carreau fluid with $\mu_{\infty}^{*}=0.1, n=0.5$ and $H_{\infty}^{*}=0.1$, for $\lambda^{*}=0.1,1,10,25$ and 100; $(\mathrm{a}, \mathrm{b})$ profile of the current $H^{*}\left(\eta^{*}\right) ;(\mathrm{c}, \mathrm{d})$ basal shear stress $\tau^{*}=\mu^{*}\left(q_{0}^{*}\right) q_{0}^{*}$. Parts (b) and (d) are enlarged plots of the region of the 'nose'. The solutions for $\lambda^{*}=0.1$ are visually indistinguishable from those for a Newtonian fluid. 

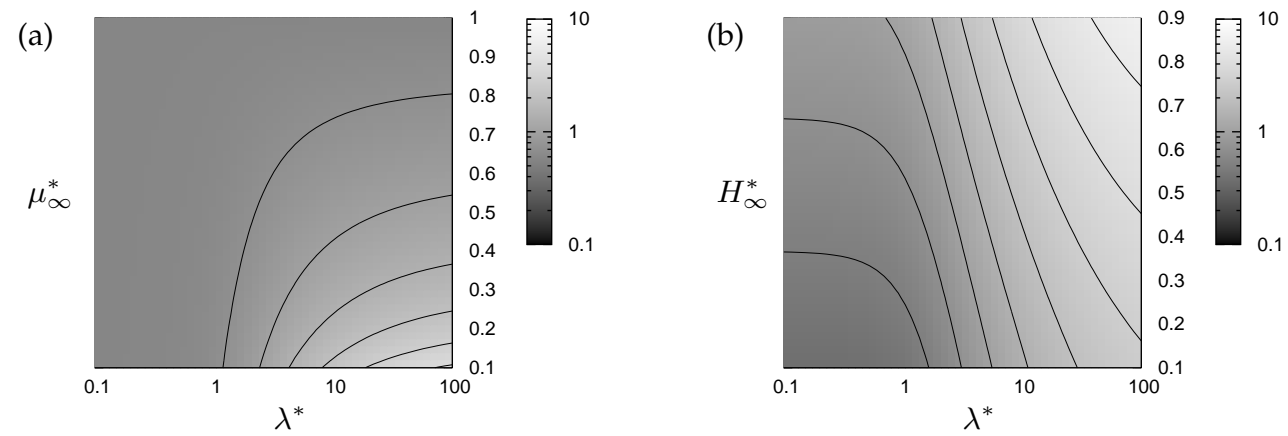

Figure 3: (a) A Carreau fluid with $n=0.5$ : the wave speed $c^{*}$ for $H_{\infty}^{*}=0.5$ as a function of the reference shear rate $\lambda^{*}$ and the high-shear-rate viscosity $\mu_{\infty}^{*}$. Contours are at $c^{*}=2^{j / 2}$ from $j=-1$ to $j=4$. (b) A Carreau fluid with $n=0.5$ and $\mu_{\infty}^{*}=0.1$ : the wave speed $c^{*}$ as a function of the reference shear rate $\lambda^{*}$ and the downstream fluid depth $H_{\infty}^{*}$. Contours are at $c^{*}=2^{j / 2}$ from $j=-2$ to $j=5$. In the Newtonian limit, $c^{*}=\left(1+H_{\infty}^{*}+H_{\infty}^{* 2}\right) / 3$.

For small values of $\lambda^{*}$, shear-thinning effects are felt only if $q_{0}^{*} \gg 1$, and so the travelling-wave solutions are very similar to those for a Newtonian fluid [29], for which

$$
\begin{array}{r}
c^{*}=\frac{1}{3}\left(1+H_{\infty}^{*}+H_{\infty}^{* 2}\right), \quad L_{0}^{*}=-\frac{1}{3} H_{\infty}^{*}\left(1+H_{\infty}^{*}\right), \\
\eta^{*}=\int \frac{H^{* 3} \mathrm{~d} H^{*}}{\left(H^{*}-H_{\infty}^{*}\right)\left(H^{*}-1\right)\left(H^{*}+H_{\infty}^{*}+1\right)} .
\end{array}
$$

As $\lambda^{*}$ increases, so the degree of shear thinning increases, its effects are felt most strongly in the region of maximum shear rate, in the middle of the 'nose' of the wave. The result of this is to reduce the peak shear stress (figure 2(d)). It also has a weak effect on the profile, tending to steepen it where the fluid is deep and make it less steep where the fluid is less deep (figures $2(a)$ and (b)). This accords with the behaviour of travelling-wave fronts in shear-thinning power-law fluids as the power-law index $n$ is varied (see, e.g., figure 6 of [29]).

Figure 3 illustrates how the wave speed $c^{*}$ varies with the rheological parameters $\lambda^{*}$ and $\mu_{\infty}^{*}$ and the depth $H_{\infty}^{*}$ of the fluid downstream. Figure 3(a) clearly shows that deviation from the Newtonian result ( $c^{*}=7 / 12 \approx 0.5833$ in this case) occurs only when $\lambda^{*}$ is large and $\mu_{\infty}^{*}$ is small: as these shear-thinning effects become stronger, the wave speed increases. The same tendency of the wave speed to increase with increasing shear-thinning (larger $\lambda^{*}$ ) is apparent in figure 3(b), which also illustrates how the wave speed increases as $H_{\infty}^{*}$ increases and the fluid layer downstream becomes more mobile.

\section{Flow on a moderately steep slope: kinematic waves}

We now consider the regime in which the slope is moderately steep, so that $|\partial h / \partial x| \ll \tan \alpha$ nearly everywhere. (We will see that this condition may be violated locally.) The hydrostatic pressure contribution to $G$ is therefore negligible; thus from (14) and (19) we have

$$
G=\rho g \sin \alpha=\text { constant }, \quad \mu\left(q_{0}\right) q_{0}=G h=\rho g \sin \alpha h .
$$

Equation (20) can thus be written as

$$
\frac{\partial h}{\partial t}+\frac{\mu\left(q_{0}\right) q_{0}^{2}}{G^{2}}\left[\mu\left(q_{0}\right) q_{0}\right]^{\prime} \frac{\partial q_{0}}{\partial x}=0
$$


Using (39b) we have

$$
\left[\mu\left(q_{0}\right) q_{0}\right]^{\prime} \frac{\partial q_{0}}{\partial x}=G \frac{\partial h}{\partial x} \quad \text { and } \quad \frac{\mu\left(q_{0}\right) q_{0}^{2}}{G^{2}}=\frac{h q_{0}}{G},
$$

and so equation (40) becomes the kinematic-wave equation

$$
\frac{\partial h}{\partial t}+c(h) \frac{\partial h}{\partial x}=0, \quad \mu\left(q_{0}\right) q_{0}=\rho g \sin \alpha h, \quad c(h)=q_{0} h=\frac{\mu\left(q_{0}\right) q_{0}^{2}}{\rho g \sin \alpha} .
$$

The implicit solution of (42) satisfying $h(x, 0)=h_{0}(x)$ for some initial free surface shape $h_{0}(x)$ is

$$
h=h_{0}(x-c(h) t),
$$

in which $c(h)$ is given implicitly by eliminating $q_{0}$ from (42).

Note that the existing results for the particular case of a power-law fluid [23] may easily be recovered. From equations (39)-(43) we have

$$
q_{0}=\left(\frac{\rho g \sin \alpha}{\mu_{n}}\right)^{1 / n} h^{1 / n}, \quad c(h)=\left(\frac{\rho g \sin \alpha}{\mu_{n}}\right)^{1 / n} h^{(n+1) / n},
$$

and thus

$$
\frac{\partial h}{\partial t}+\left(\frac{\rho g \sin \alpha}{\mu_{n}}\right)^{1 / n} h^{(n+1) / n} \frac{\partial h}{\partial x}=0, \quad h=h_{0}\left(x-\left(\frac{\rho g \sin \alpha}{\mu_{n}}\right)^{1 / n} h^{(n+1) / n} t\right) .
$$

If the function $z=h_{0}(x)$ has inverse $x=h_{0}^{-1}(z)$ on some suitable domain then the solution (42) and (43) may be written

$$
x=c(h) t+h_{0}^{-1}(h), \quad \mu\left(q_{0}\right) q_{0}=\rho g \sin \alpha h,
$$

which with $c(h)=q_{0} h=\mu\left(q_{0}\right) q_{0}^{2} / \rho g \sin \alpha$ leads to a parametric representation for $h$ at time $t$ in terms of the parameter $q_{0}$, namely

$$
x=\frac{\mu\left(q_{0}\right) q_{0}^{2}}{\rho g \sin \alpha} t+h_{0}^{-1}\left(\frac{\mu\left(q_{0}\right) q_{0}}{\rho g \sin \alpha}\right), \quad h=\frac{\mu\left(q_{0}\right) q_{0}}{\rho g \sin \alpha} .
$$

As is common with kinematic waves, this general solution is too general to be directly informative, and so we will specialise further to a particularly relevant class of solutions.

\subsection{A finite release of fluid: general solution at finite $t$}

Consider the release at time $t=0$ of a fixed quantity of fluid, with finite length in the $x$ direction and with volume $A$ (per unit width in the $y$ direction). The mass of fluid is constant, and so

$$
\int_{0}^{x_{\mathrm{F}}(t)} h(x, t) \mathrm{d} x=A
$$

at all time, where $x=x_{\mathrm{F}}(t)$ denotes the position of the front of the fluid at time $t$. Equation (48) is an algebraic equation which determines $x_{F}$; the solution for $h$ is then given by (43) for $0 \leq x \leq x_{\mathrm{F}}$ 


\subsection{A finite release of fluid: centred-wave solution at large $t$}

In the asymptotic limit of large time, $t \rightarrow \infty$, the current 'forgets' its initial state [8], and the solution (43) for $h$ takes the form of a centred wave, for which

$$
c(h) \sim \frac{x}{t} .
$$

This shows that, no matter what form the function $\mu(q)$ may take, eventually $h$ depends on $x$ and $t$ only in the combination $x / t$. Moreover, at large $t$ equations (42) and (49) give $h$ parametrically in terms of $q_{0}$ :

$$
h=\frac{\mu\left(q_{0}\right) q_{0}}{\rho g \sin \alpha}, \quad \frac{x}{t} \sim \frac{\mu\left(q_{0}\right) q_{0}^{2}}{\rho g \sin \alpha} .
$$

Since also $c=q_{0} h$, equation (49) or (50) shows that $q_{0} \sim x /(h t)$ at large $t$, and so the parametric solution (50) may alternatively be written in the implicit form

$$
\mu\left(\frac{x}{h t}\right)\left(\frac{x}{h t}\right)^{2} \sim \rho g \sin \alpha \frac{x}{t} .
$$

For the centred-wave solution (50) the mass-conservation condition (48) leads to

$$
(\rho g \sin \alpha)^{2} A=t \int_{0}^{q_{0 \mathrm{~F}}} \mu\left(q_{0}\right) q_{0} \frac{\mathrm{d}\left[\mu\left(q_{0}\right) q_{0}^{2}\right]}{\mathrm{d} q_{0}} \mathrm{~d} q_{0}=t\left[\mu\left(q_{0 \mathrm{~F}}(t)\right)\right]^{2} q_{0 \mathrm{~F}}^{3}(t)-t F\left(q_{0 \mathrm{~F}}(t)\right),
$$

where $q_{0 \mathrm{~F}}(t)=q_{0}\left(x_{\mathrm{F}}(t), t\right)$ is the shear rate at the substrate at the front $x=x_{\mathrm{F}}(t)$. (Note that the first term on the right-hand side of (52) may be written as $(\rho g \sin \alpha)^{2} x_{\mathrm{F}} h_{\mathrm{F}}$, illustrating how the rheology of the fluid, represented by $F\left(q_{0}\right)$, 'corrects' the shape of the current.) Equation (52) is an algebraic equation which determines $q_{0 \mathrm{~F}}(t)$; the front position and the depth at the front $h_{\mathrm{F}}(t) \equiv h\left(x_{\mathrm{F}}(t), t\right)$ are then given by

$$
h_{\mathrm{F}}(t)=\frac{\mu\left(q_{0 \mathrm{~F}}\right) q_{0 \mathrm{~F}}}{\rho g \sin \alpha}, \quad x_{\mathrm{F}}(t)=\frac{\mu\left(q_{0 \mathrm{~F}}\right) q_{0 \mathrm{~F}}^{2}}{\rho g \sin \alpha} t
$$

at large $t$. Note that this solution has a height discontinuity at the front, where the condition $\left|\cos \alpha h_{x}\right| \ll \sin \alpha$ breaks down. In a solution of the full evolution equation (20), a steep but continuous 'nose' may be expected to replace this discontinuity, and a matched asymptotic expansion may be constructed to describe it (e.g. [10, 25]); however, the advance of the current and its profile away from the nose are still described to leading order by (53).

We can again easily recover the existing results for a power-law fluid, which have recently been presented by Ganguly et al. [24]. When $c(h)$ is given by (44), equation (50) gives

$$
h=\frac{\mu_{n} q_{0}^{n}}{\rho g \sin \alpha}, \quad \frac{x}{t} \sim \frac{\mu_{n} q_{0}^{n+1}}{\rho g \sin \alpha},
$$

leading to

$$
h \sim\left(\frac{\mu_{n}}{\rho g \sin \alpha}\right)^{1 /(n+1)}\left(\frac{x}{t}\right)^{n /(n+1)} \quad \text { as } \quad t \rightarrow \infty,
$$

while equation (52) gives

$$
(\rho g \sin \alpha)^{2} A=(n+1) \mu_{n}^{2} t \int_{0}^{q_{0 \mathrm{~F}}} q_{0}^{2 n} \mathrm{~d} q_{0}=\left(\frac{n+1}{2 n+1}\right) \mu_{n}^{2} q_{0 \mathrm{~F}}^{2 n+1} t,
$$

and so

$$
q_{0 \mathrm{~F}}=\left(\frac{(2 n+1)(\rho g \sin \alpha)^{2} A}{(n+1) \mu_{n}^{2} t}\right)^{1 /(2 n+1)} .
$$


Hence from (53) we recover the solution

$$
h_{\mathrm{F}}(t)=\left[\left(\frac{2 n+1}{n+1}\right)^{n} \frac{\mu_{n} A^{n}}{\rho g \sin \alpha t^{n}}\right]^{1 /(2 n+1)}, \quad x_{\mathrm{F}}(t)=\left[\left(\frac{2 n+1}{n+1}\right)^{n+1} \frac{\rho g \sin \alpha A^{n+1} t^{n}}{\mu_{n}}\right]^{1 /(2 n+1)}
$$

as $t \rightarrow \infty$. Like many self-similar solutions, the form of (58), although not the dimensionless prefactors, may be deduced from purely dimensional considerations. (Equation (58) shows, incidentally, that $h_{\mathrm{F}} x_{\mathrm{F}}=((2 n+1) /(n+1)) A=$ constant. This reflects the fact that these solutions are self-similar; we shall show below that it is not necessarily the case for other rheologies.)

It is straightforward to generalise the above solutions to the case when fluid is supplied or removed in a prescribed fashion, so that the area $A$ is time-dependent; for brevity we omit this generalisation here.

\subsubsection{Centred-wave solution for a Carreau fluid}

To demonstrate the applicability of our approach to more complicated rheologies, we now construct centred-wave solutions for the case of a Carreau fluid with constitutive equation (28). Before presenting the results, it is convenient to non-dimensionalise the variables appropriately. We define dimensionless quantities, denoted by an asterisk, via

$$
q_{0}=\frac{q^{*}}{\lambda}, t=\left(\frac{\rho g \sin \alpha}{\mu_{0}}\right)^{2} A \lambda^{3} t^{*}, x=\frac{\rho g \sin \alpha A \lambda}{\mu_{0}} x^{*}, h=\frac{\mu_{0} h^{*}}{\rho g \sin \alpha \lambda}, \mu(q)=\mu_{0} \mu^{*}\left(q^{*}\right) .
$$

We can then write the dimensionless viscosity function as

$$
\mu^{*}\left(q^{*}\right)=\mu_{\infty}^{*}+\frac{1-\mu_{\infty}^{*}}{\left(1+q^{* 2}\right)^{(1-n) / 2}}, \quad \text { where } \quad \mu_{\infty}^{*}=\frac{\mu_{\infty}}{\mu_{0}},
$$

and the centred kinematic-wave solution parameterised by $q^{*}$ becomes

$$
h^{*}=\mu^{*}\left(q^{*}\right) q^{*}, \quad \frac{x^{*}}{t^{*}} \sim \mu^{*}\left(q^{*}\right) q^{* 2}, \quad 1=t^{*} \int_{0}^{q_{0 \mathrm{~F}}^{*}} \mu^{*}\left(q^{*}\right) q^{*} \frac{\mathrm{d}\left[\mu^{*}\left(q^{*}\right) q^{* 2}\right]}{\mathrm{d} q^{*}} \mathrm{~d} q^{*} .
$$

As in section 3.1, the parameter $\mu_{\infty}^{*}$ determines whether the fluid is shear-thinning $\left(\mu_{\infty}^{*}<1\right)$ or shear-thickening $\left(\mu_{\infty}^{*}>1\right)$. Note that the characteristic shear rate $1 / \lambda$ in the viscosity function sets the time- and lengthscales for the evolution of the flow. Where the shear rate is low, $q^{*} \ll 1$, the viscosity tends to its low-shear-rate value and the fluid behaves like a Newtonian fluid with constant dimensionless viscosity 1 . Where the shear rate is high, $q^{*} \gg 1$, the viscosity tends to its high-shear-rate value and the fluid behaves like a Newtonian fluid with constant dimensionless viscosity $\mu_{\infty}^{*}$. The parameter $n$ determines how rapidly the viscosity changes between its low- and high-shear values; in contrast to the travelling waves considered in section 3.1, the kinematic-wave solutions are somewhat sensitive to the value of $n$.

Figures 4 and 5 illustrate the behaviour of the solutions for a shear-thinning Carreau fluid $\left(\mu_{\infty}^{*}=0.2\right)$ for three values of $n$; results for shear-thickening fluids are analogous and are omitted here for brevity. As the current spreads and thins, both the depth at the front, $h_{\mathrm{F}}^{*}\left(t^{*}\right)$, and the front velocity, $\mathrm{d} x_{\mathrm{F}}^{*} / \mathrm{d} t^{*}$, decrease (figures $4(\mathrm{~b})$ and (c)); the net effect is that the shear rate at the front, $q_{0 \mathrm{~F}}^{*}$, decreases (figure $4(\mathrm{a})$ ). At sufficiently early times, the shear rate at the front is high, $q_{0 \mathrm{~F}}^{*} \gg 1$, and so the fluid behaves like a Newtonian fluid with dimensionless viscosity $\mu^{*}=0.2$. In this regime, we have

$$
q_{0 \mathrm{~F}}^{*} \sim\left(\frac{3}{2}\right)^{1 / 3} \mu_{\infty}^{*-2 / 3} t^{*-1 / 3}, \quad x_{\mathrm{F}}^{*} \sim\left(\frac{3}{2}\right)^{2 / 3} \mu_{\infty}^{*-1 / 3} t^{* 1 / 3} \quad \text { and } \quad h_{\mathrm{F}}^{*} \sim\left(\frac{3}{2}\right)^{1 / 3} \mu_{\infty}^{* 1 / 3} t^{*-1 / 3},
$$


(a)

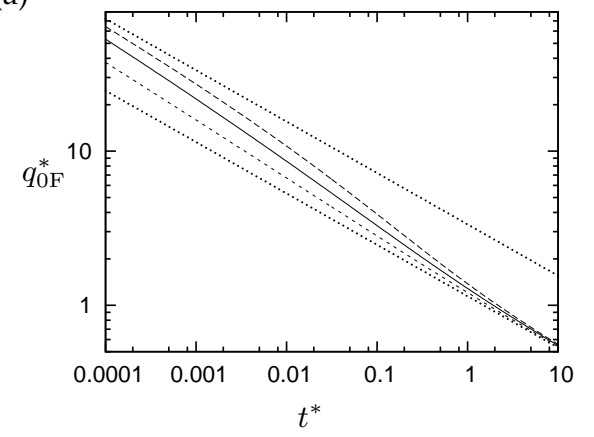

(c)

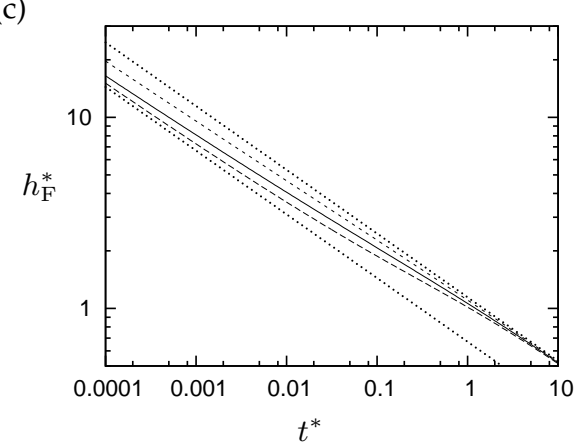

(b)

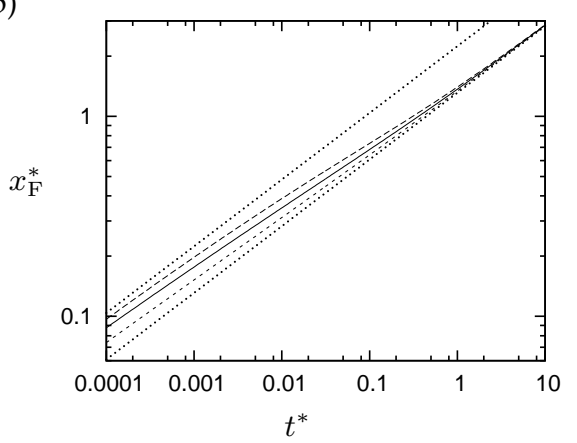

Figure 4: Centred-wave solution: quantities at the front of the current for a Carreau fluid with $\mu_{\infty}^{*}=0.2$ and $n=0.5$ (solid), $n=0.25$ (heavy dashed), $n=0.75$ (light dashed) : (a) front shear rate $q_{0 \mathrm{~F}}^{*}\left(t^{*}\right)$; (b) front position $x_{\mathrm{F}}^{*}\left(t^{*}\right)$; (c) depth at the front $h_{\mathrm{F}}^{*}\left(t^{*}\right)$. Dotted lines in each plot show the results for Newtonian fluids with $\mu^{*}=1$ and with $\mu^{*}=0.2$. Note the logarithmic scales in each case. 
(a)

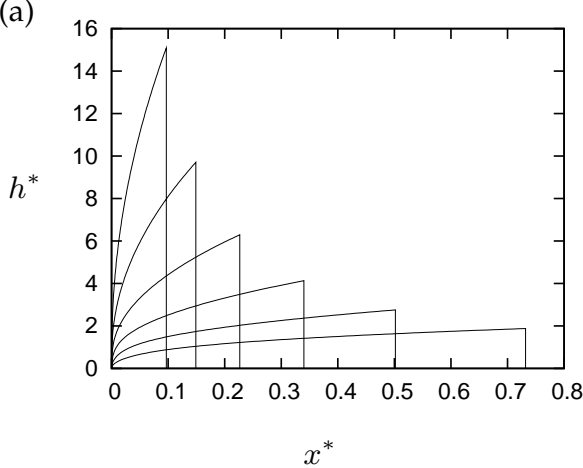

(c)

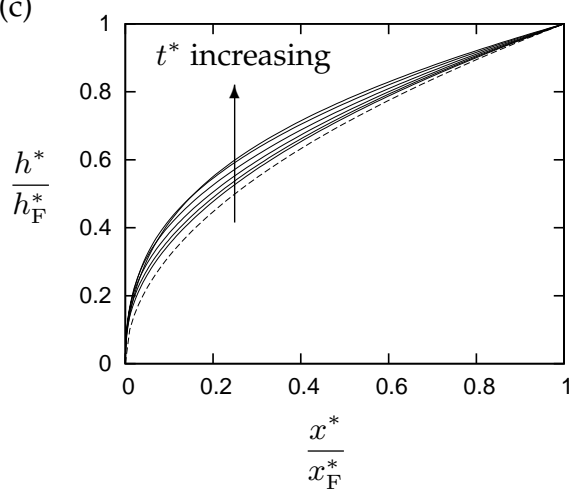

(b)

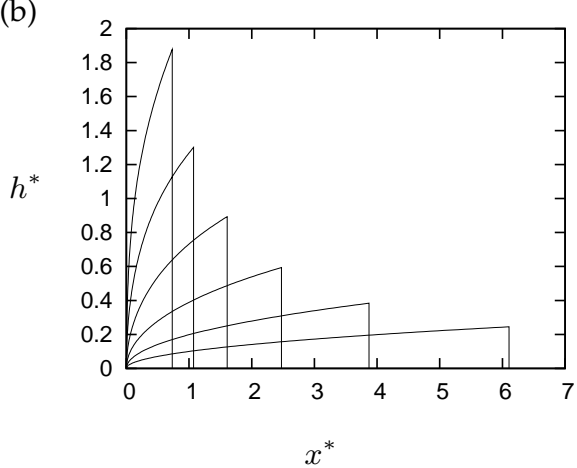

(d)

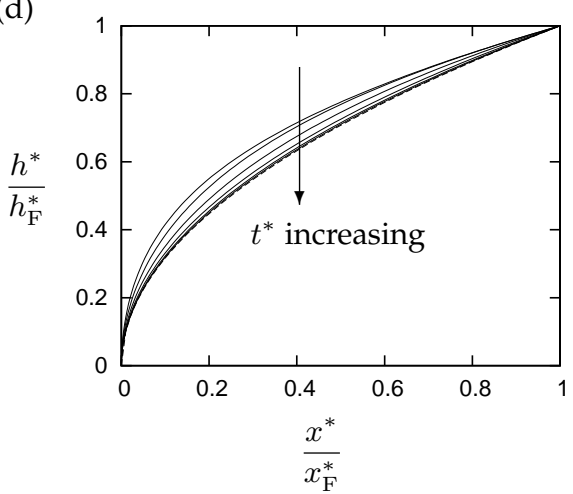

Figure 5: Profiles of the centred-wave solution for a Carreau fluid with $\mu_{\infty}^{*}=0.2$ and $n=$ 0.25: (a) and (b) show profiles $h^{*}$ at various times plotted as functions of the space variable $x^{*}$, while (c) and (d) show scaled profiles $h^{*} / h_{\mathrm{F}}^{*}$ at the same times, plotted as functions of the scaled space variable $x^{*} / x_{\mathrm{F}}^{*}$; the dashed line in these figures is the Newtonian solution $h^{*} / h_{\mathrm{F}}^{*}=\left(x^{*} / x_{\mathrm{F}}^{*}\right)^{1 / 2}$. Figures (a) and (c) show profiles at early times, from $t^{*}=0.0001$ to $t^{*}=0.1$, for six exponentially spaced values of $t^{*}$. Figures (b) and (d) show profiles at later times, from $t^{*}=0.1$ to $t^{*}=100$, for six exponentially spaced values of $t^{*}$.

from equations (58) with $n=1$. As the current evolves and $q_{0 \mathrm{~F}}^{*}$ decreases, the non-Newtonian effects become apparent, and at around $t^{*} \sim 1$ there is a gradual transition to a low-shear-rate regime in which $q_{0 \mathrm{~F}}^{*} \ll 1$ and so $\mu^{*} \sim 1$. In this low-shear-rate regime, the Newtonian scalings are again recovered, but with different coefficients of proportionality:

$$
q_{0 \mathrm{~F}}^{*} \sim\left(\frac{3}{2}\right)^{1 / 3} t^{*-1 / 3}, \quad x_{\mathrm{F}}^{*} \sim\left(\frac{3}{2}\right)^{2 / 3} t^{* 1 / 3} \quad \text { and } \quad h_{\mathrm{F}}^{*} \sim\left(\frac{3}{2}\right)^{1 / 3} t^{*-1 / 3} .
$$

Although the same transition is seen for each value of $n$, it occurs significantly earlier for higher values of $n$, for which the denominator $\left(1+q^{* 2}\right)^{(1-n) / 2}$ is smaller and so the viscosity is closer to $\mu_{\infty}^{*}$ for a given value of $q^{*}$.

The transition from high- to low-shear-rate behaviour can also be seen in the changing profile $h^{*}\left(x^{*}, t^{*}\right)$ of the current, which goes through two phases of adjustment (figure 5). At early times, $t^{*} \ll 1$, the shear rate is high everywhere and the profile is reasonably close to the Newtonian one (figure 5(c)), deviating most from it near the tail of the current where the shear rate is always low (since, from (50), $q_{0}=0$ when $x / t=0$ ). As $t^{*}$ increases and the flow decelerates, the low-shear-rate region expands forward, and so in figure 5(c) we see an adjustment in the profile propagating forward from the tail, eventually catching up with the flow front at around $t^{*} \approx 0.1$. Thereafter, the profile gradually adjusts back to its Newtonian form (figure 5(d)). 
It is worth remarking that although the solution depends on $x^{*}$ and $t^{*}$ only through the combination $x^{*} / t^{*}$, the front position $x_{\mathrm{F}}^{*}\left(t^{*}\right)$ does not correspond to a fixed value of $x^{*} / t^{*}$ and so the solutions are not perfectly self-similar. Consequently the normalised profiles in figures 5(c) and (d) do not collapse onto a single curve. This contrasts with the solutions for power-law fluids (including the Newtonian case), in which $h^{*}$ is proportional to a simple power of $x^{*}$; regardless of the variation of $x_{\mathrm{F}}^{*}\left(t^{*}\right)$, the normalised profiles $h^{*} / h_{\mathrm{F}}^{*}$ for such solutions must always collapse onto a single curve when plotted as functions of $x^{*} / x_{\mathrm{F}}^{*}$. It is straightforward to show from (50) that this property can hold only when $\mu\left(q_{0}\right)$ is a simple power of $q_{0}$.

\subsubsection{Centred-wave solutions for yield-stress fluids}

The approach outlined above, in which solutions are written in terms of the shear rate $q$, relies on making the change of variables from $z$ to $q$. Consequently, it is not applicable to fluids with a yield stress $\tau_{\mathrm{y}}$, for which $q=0$ throughout the pseudo-plug region near the free surface where $0 \leq \tau<\tau_{\mathrm{y}}$ [15]. However, from equation (15) the shear stress $\tau=\mu(q) q$ varies monotonically with $z$; consequently, the formulation (21) in terms of $\tau$ is applicable to yield-stress fluids. An advantage of this formulation is that it is no longer necessary to keep track explicitly of the yield surface. We will illustrate the approach (21) here by obtaining solutions for kinematic waves first in general terms and then for the Bingham and Casson rheologies [38, §1.5]; the former solutions are specialisations of those due to Huang \& Garcia [25], while the latter appear to be new.

On a moderately steep slope, we again have

$$
\tau_{0}=G h, \quad \text { where } \quad G=\rho g \sin \alpha,
$$

and the evolution equation (21) becomes

$$
\frac{\partial h}{\partial t}+c(h) \frac{\partial h}{\partial x}=0, \quad \text { where } \quad c(h)=h \phi(G h) .
$$

For suitable initial conditions, equation (49) again provides the implicit solution at large times. Suitable initial conditions are required in this case because a yield-stress fluid on a slope is mobile only when $h$ exceeds the 'yield depth' $h_{\mathrm{y}}=\tau_{\mathrm{y}} / G$ below which $\phi(G h)=0$; where $h<h_{\mathrm{y}}$, the fluid cannot flow downslope under its own weight. Thus there is a maximum run-out distance $x_{\infty}=A / h_{\mathrm{y}}$, and (49) provides a good approximation at large times only if the initial distribution of fluid occupied a length much less than $x_{\infty}$.

The volume conservation condition can be written in terms of an integral either over $h$ or over $\tau_{0}$ :

$$
A=\int_{0}^{x_{\mathrm{F}}(t)} h(x, t) \mathrm{d} x=t \int_{h_{\mathrm{y}}}^{h_{\mathrm{F}}} h \frac{\mathrm{d} c}{\mathrm{~d} h} \mathrm{~d} h=\frac{t}{G^{2}} \int_{\tau_{\mathrm{y}}}^{\tau_{0 \mathrm{~F}}} \tau_{0} \frac{\mathrm{d}}{\mathrm{d} \tau_{0}}\left[\tau_{0} \phi\left(\tau_{0}\right)\right] \mathrm{d} \tau_{0} .
$$

This condition implicitly defines $h_{\mathrm{F}}$ and $\tau_{0 \mathrm{~F}}$, respectively the depth and the basal shear stress at the flow front, and hence the position of the front via $c\left(h_{\mathrm{F}}\right)=x_{\mathrm{F}} / t$.

For the simple case of a Bingham fluid with high-shear-rate viscosity $\mu_{B}$ and yield stress $\tau_{\mathrm{y}}$, in simple shearing flow we have

$$
q=\phi(\tau)= \begin{cases}\frac{1}{\mu_{B}}\left(\tau-\tau_{\mathrm{y}}\right) & \text { if } \tau>\tau_{\mathrm{y}} \\ 0 & \text { otherwise }\end{cases}
$$

The kinematic wave speed $c(h)$ may then be written as

$$
c(h)= \begin{cases}\frac{G}{\mu_{B}} h\left(h-h_{\mathrm{y}}\right) & \text { if } h>h_{\mathrm{y}}, \\ 0 & \text { otherwise. }\end{cases}
$$


The volume condition (66) becomes

$$
\frac{A}{t}=\int_{h_{\mathrm{y}}}^{h_{\mathrm{F}}} h \frac{\mathrm{d} c}{\mathrm{~d} h} \mathrm{~d} h=\frac{G}{\mu_{B}}\left(\frac{2}{3} h_{\mathrm{F}}^{3}-\frac{1}{2} h_{\mathrm{y}} h_{\mathrm{F}}^{2}-\frac{1}{6} h_{\mathrm{y}}^{3}\right) .
$$

For the slightly less simple case of a Casson fluid with high-shear-rate viscosity $\mu_{C}$ and yield stress $\tau_{\mathrm{y}}$, in simple shear we have

$$
q=\phi(\tau)= \begin{cases}\frac{1}{\mu_{C}}\left(\tau^{1 / 2}-\tau_{\mathrm{y}}^{1 / 2}\right)^{2} & \text { if } \tau>\tau_{\mathrm{y}} \\ 0 & \text { otherwise }\end{cases}
$$

This yields the kinematic wave speed

$$
c(h)= \begin{cases}\frac{G}{\mu_{C}} h\left(h^{1 / 2}-h_{\mathrm{y}}^{1 / 2}\right)^{2} & \text { if } h>h_{\mathrm{y}}, \\ 0 & \text { otherwise }\end{cases}
$$

and the global volume condition

$$
\frac{A}{t}=\frac{G}{\mu_{C}} \int_{h_{\mathrm{y}}}^{h_{\mathrm{F}}} h\left(2 h-3 \sqrt{h h_{\mathrm{y}}}+h_{\mathrm{y}}\right) \mathrm{d} h=\frac{G}{\mu_{C}}\left(\frac{2}{3} h_{\mathrm{F}}^{3}-\frac{6}{5} \sqrt{h_{\mathrm{y}}} h_{\mathrm{F}}^{5 / 2}+\frac{1}{2} h_{\mathrm{y}} h_{\mathrm{F}}^{2}+\frac{1}{30} h_{\mathrm{y}}^{3}\right) .
$$

We will compare the results for Bingham and Casson fluids with $\mu_{B}=\mu_{C}=\mu$ and identical values of $\tau_{\mathrm{y}}$. We define dimensionless variables, denoted by an asterisk, via

$$
h=h_{\mathrm{y}} h^{*}, \quad x=x_{\infty} x^{*}=\frac{A}{h_{\mathrm{y}}} x^{*} \quad \text { and } \quad t=\frac{A \mu}{G h_{\mathrm{y}}^{3}} t^{*} .
$$

The centred-wave solution for a Bingham fluid, defined by (49), (68) and (69), becomes

$$
\frac{x^{*}}{t^{*}}=h^{*}\left(h^{*}-1\right) \quad \text { for } 1 \leq h^{*} \leq h_{\mathrm{F}}^{*}, \quad \text { where } \quad \frac{2}{3} h_{\mathrm{F}}^{* 3}-\frac{1}{2} h_{\mathrm{F}}^{* 2}-\frac{1}{6}=\frac{1}{t^{*}} .
$$

while the centred-wave solution for a Casson fluid, defined by (49), (71) and (72), becomes

$$
\frac{x^{*}}{t^{*}}=h^{*}\left(h^{* 1 / 2}-1\right)^{2} \quad \text { for } \quad 1 \leq h^{*} \leq h_{\mathrm{F}}^{*}, \quad \text { where } \quad \frac{2}{3} h_{\mathrm{F}}^{* 3}-\frac{6}{5} h_{\mathrm{F}}^{* 5 / 2}+\frac{1}{2} h_{\mathrm{F}}^{* 2}+\frac{1}{30}=\frac{1}{t^{*}} .
$$

Figures 6 and 7 illustrate the solutions (74) and (75): figure 6 shows how the length and the depth at the front of the current evolve, while figure 7 illustrates the evolving profile of the current.

For a given shear rate $q>0$, the Casson model predicts a higher shear stress $\tau$ than does the Bingham model with the same yield stress and high-shear-rate viscosity; informally, the Casson fluid is more viscous at finite shear rates than the Bingham fluid. A consequence of this is that the Casson fluid advances more slowly towards its ultimate run-out length $x_{\mathrm{F}}^{*}=1$ than the Bingham fluid (figure 6(a)), and the depth at the front decreases more slowly (figure $6(b))$.

These trends are the most visible feature when the current profiles are plotted for successive times (figure 7). It is also apparent in these plots that as $t^{*} \rightarrow \infty$ the current evolves towards a static deposit with a constant depth $h^{*}=1$, corresponding to $h=h_{\mathrm{y}}$. Although the runout distance is finite, it is not attained in a finite time: this is perhaps most clearly seen by noting that as $h_{F} \rightarrow 1$, the left-hand sides of equations (74b) and (75b) tend to zero. (Note that the yield surface, not plotted here, lies a constant dimensionless distance of 1 below the free surface when $h^{*} \geq 1$.) At earlier times, the profile of the Casson fluid (figure 7(c)) varies more strongly from this rectangular limiting case than does that of the Bingham fluid (figure 7(d)), but the difference is rather subtle and would not easily be identified experimentally. 
(c)

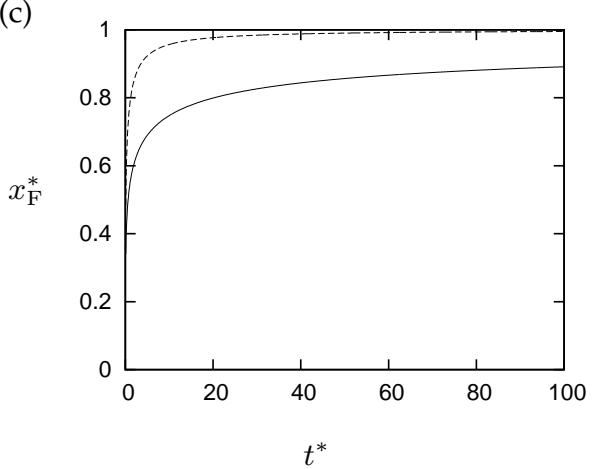

(d)

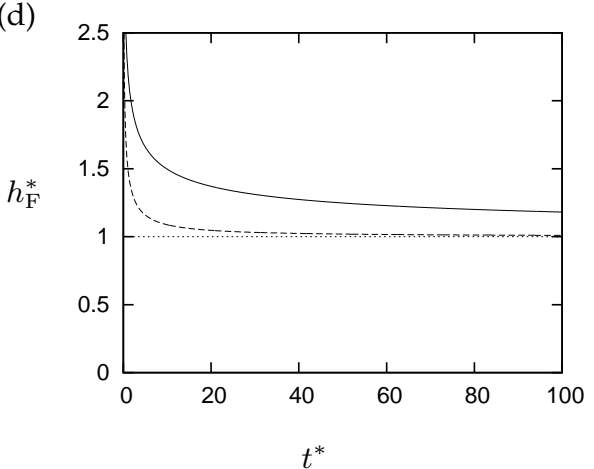

Figure 6: Comparison of the centred-wave solutions for a Casson fluid (solid lines) and a Bingham fluid (dashed lines): (a) the length of the current $x_{\mathrm{F}}^{*}$; (b) the depth at the front of the current $h_{\mathrm{F}}^{*}$, with the large-time asymptotic limit $h_{\mathrm{F}}^{*}=1$ (dotted line).

(a)

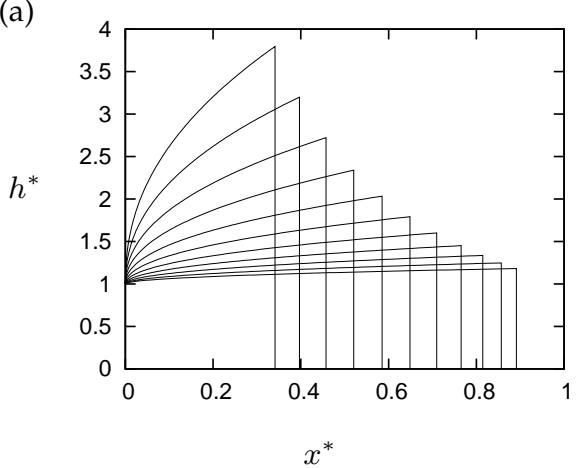

(c)

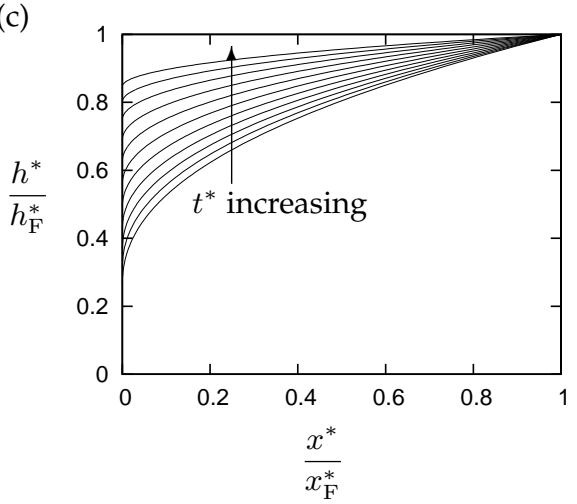

(b)

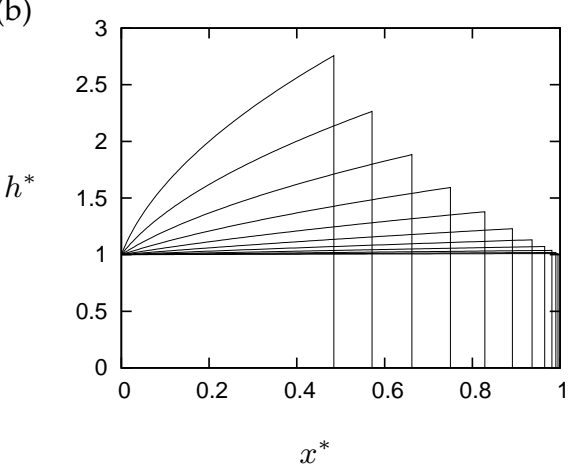

(d)

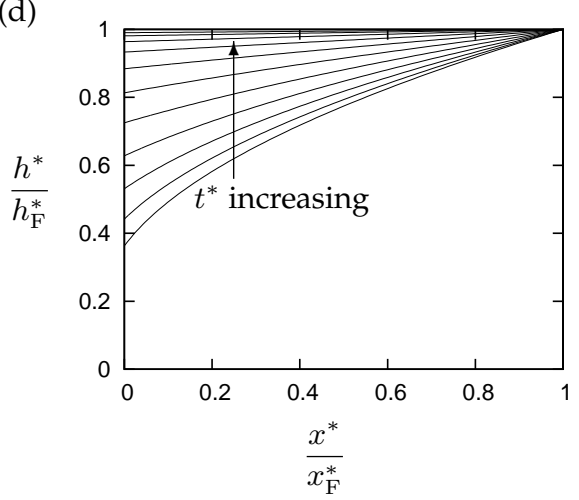

Figure 7: Profiles of the centred-wave solution for (a), (c) a Casson fluid and (b), (d) a Bingham fluid: (a) and (b) show profiles $h^{*}$ at various times plotted as functions of the space variable $x^{*}$, while (c) and (d) show scaled profiles $h^{*} / h_{\mathrm{F}}^{*}$ at the same times, plotted as functions of the scaled space variable $x^{*} / x_{\mathrm{F}}^{*}$. All profiles are plotted at eleven exponentially spaced values of $t^{*}$ from $t^{*}=0.1$ to $t^{*}=100$.

\section{Summary and conclusions}

We have derived a general evolution equation for a shallow layer of generalised Newtonian fluid undergoing unsteady two-dimensional gravity-driven flow on a horizontal or an inclined 
plane. The variables in this equation are the depth $h(x, t)$ of the layer and an additional variable $q_{0}(x, t)$ or $\tau_{0}(x, t)$ which represents the basal shear rate or shear stress and which is related to $h$ and its derivatives by an implicit equation. In certain regimes of the slope, this evolution equation reduces further, and we have presented two classes of solutions, illustrating the results for particular choices of rheology.

For flows on mild slopes, we have presented novel travelling-wave solutions for Carreau fluids, investigating the effect of shear thinning on the solutions. The overall character of the solutions is rather insensitive to the rheology, but shear thinning reduces the peak of shear stress at the wave front, and also tends to increase the wave speed. For flows on moderately steep slopes, we have presented novel centred kinematic-wave solutions for Carreau and Casson fluids, comparing the latter with previously derived results for Bingham fluids [25]. The principal feature of the solutions for Carreau fluids is a transition from high-shear-rate behaviour at early times to low-shear-rate behaviour at later times; it is also noteworthy that, unlike the solutions for power-law fluids, solutions for other rheologies are not, in general, self-similar. An interesting feature of the solutions for yield-stress fluids is that although a Bingham fluid and a Casson fluid with the same yield stress and high-shear-rate viscosity run out to the same distance, the run-out of the Bingham fluid is considerably faster because it is less viscous at intermediate shear rates.

Although we have presented solutions for specific rheological models, we stress that the same procedure can be applied for any generalised Newtonian fluid; crucially, it does not require one to obtain the velocity profile in the flow explicitly. In particular, the version of the procedure which employs integration over the shear stress offers a means of obtaining shallowflow solutions for yield-stress fluids without explicitly tracking the yield surface within the fluid. The approach presented here therefore offers a useful way to develop test-bed solutions with which to validate numerical methods for free-surface non-Newtonian flows. This approach also offers a means by which theoretical predictions may be obtained to compare with experimental results for which simple constitutive laws are known to be inappropriate, for example allowing recent work for the Herschel-Bulkley model [35] to be extended to other rheologies. Another promising direction for the further development of this approach is to flows for which variation in both the downslope and transverse directions is important, such as non-Newtonian rivulets $[42,43,44]$.

Acknowledgements. This work was begun while S. K. W. was a Visiting Fellow at the Isaac Newton Institute for Mathematical Sciences in Cambridge, England, U. K., as part of the programme on 'Mathematical Modelling and Analysis of Complex Fluids and Active Media in Evolving Domains', and finished while he was a Leverhulme Trust Research Fellow (20132015) supported by award RF-2015-355, 'Small Particles, Big Problems: Understanding the Complex Behaviour of Nanofluids'.

\section{References}

[1] Reynolds O (1886) On the theory of lubrication and its application to Mr. Beauchamp Tower's experiments, including an experimental demonstration of the viscosity of olive oil. Phil Trans Roy Soc London 177:157-234

[2] Oron A, Davis SH, Bankoff SG (1997) Long-scale evolution of thin liquid films. Rev Mod Phys 69:931-980

[3] Craster RV, Matar OK (2009) Dynamics and stability of thin liquid films. Rev Mod Phys 81:1131-1198 
[4] Ancey C (2001) Debris flows and related phenomena. In: Balmforth NJ, Provenzale A (eds), Geomorphological Fluid Mechanics, Springer, chapter 21, pp 528-547

[5] Huppert HE (1982a) The propagation of two-dimensional and axisymmetric viscous gravity currents over a rigid horizontal surface. J Fluid Mech 121:43-58

[6] Huppert HE (1982b) Flow and instability of a viscous current down a slope. Nature 300:427-429

[7] Whitham GB (1974) Linear and Nonlinear Waves. Wiley-Interscience

[8] Weir GJ (1983) The asymptotic behaviour of simple kinematic waves of finite volume. Proc Roy Soc London A 387:459-467

[9] Troian SM, Herbolzheimer E, Safran SA, Joanny JF (1989) Fingering instabilities of driven spreading films. Europhys Lett 10:25-30

[10] Hunt B (1994) Newtonian fluid mechanics treatment of debris flows and avalanches. J Hydraul Eng 120:1350-1363

[11] Balmforth NJ, Craster RV (2001) Geophysical aspects of non-Newtonian fluid mechanics. In: Balmforth NJ, Provenzale A (eds), Geomorphological Fluid Mechanics, Springer, chapter 2, pp 34-51

[12] Gorodtsov VA (1989) Spreading of a film of nonlinearly viscous liquid over a horizontal smooth surface. J Eng Phys Thermophys 57:879-884. Tr. from Inzherno-Fizicheskii Zhurnal 57(2): 203-209, August 1989.

[13] Pascal H (1991) Gravity flow of a non-Newtonian fluid sheet on an inclined plane. Int J Eng Sci 29:1307-1313

[14] Gratton J, Minotti F, Mahajan SA (1999) Theory of creeping gravity currents of a nonNewtonian liquid. Phys Rev E 60:6960-6967

[15] Balmforth NJ, Craster RV (1999) A consistent thin-layer theory for Bingham plastics. J Non-Newton Fluid Mech 84:65-81

[16] Balmforth NJ, Burbidge AS, Craster RV, Salzig J, Shen A (2000) Visco-plastic models of isothermal lava domes. J Fluid Mech 403:37-65

[17] Ross AB, Wilson SK, and Duffy BR (2001) Thin-film flow of a viscoplastic material round a large horizontal stationary or rotating cylinder. J Fluid Mech 430:309-333

[18] Nye JF (1960) The response of glaciers and ice-sheets to seasonal and climatic changes. Proc Roy Soc London A 256(1287):559-584

[19] Fowler AC, Larson DA (1978) On the flow of polythermal glaciers. I. Model and preliminary analysis. Proc Roy Soc London A 363(1713):217-242

[20] Hutter K (1980) Time-dependent surface elevation of an ice slope. J Glaciol 25:247-266

[21] Ahlkrona J, Kirchner N, Lötstedt P (2013) A numerical study of scaling relations for nonNewtonian thin-film flows with applications in ice sheet modelling. Q J Mech Appl Math $66: 417-435$

[22] Berezhin YuA, Hutter K, Spodareva LA (1998) Stability analysis of gravity driven shear flows with free surface for power-law fluids. Arch Appl Mech 68:169-178 
[23] Perazzo CA, Gratton J (2003) Thin film of non-Newtonian fluid on an incline. Phys Rev E 67:016307

[24] Ganguly A, Reza, M, Gupta AS (2012) Thin-film flow of a power-law fluid down an inclined plane. J Fluids Eng 134:044502-1-5

[25] Huang X, Garcia MH (1998) A Herschel-Bulkley model for mud flow down a slope. J Fluid Mech 374:305-333

[26] Ancey C, Andreini N, Epely-Chauvin G (2012) Viscoplastic dambreak waves: review of simple computational approaches and comparison with experiments. Adv Wat Res 48:79-91

[27] Kheyfets VO, Kieweg SL (2013) Gravity-driven thin film flow of an Ellis fluid. J NonNewton Fluid Mech 202:88-98

[28] Liu KF, Mei CC (1989) Slow spreading of a sheet of Bingham fluid on an inclined plane. J Fluid Mech 207:505-529

[29] Perazzo CA, Gratton J (2004) Steady and travelling flows of a power-law fluid over an incline. J Non-Newton Fluid Mech 118:57-64

[30] Chambon G, Ghemmour A, Laigle D (2009) Gravity-driven surges of a viscoplastic fluid: an experimental study. J Non-Newton Fluid Mech 158:54-62

[31] Ng C-O, Mei CC (1994) Roll waves on a shallow layer of mud modelled as a power-law fluid. J Fluid Mech 263:151-183

[32] Liu KF, Mei CC (1994) Roll waves on a layer of a muddy fluid flowing down a gentle slope - a Bingham model. Phys Fluids 6:2577-2590

[33] Laigle D, Coussot P (1997) Numerical modelling of mudflows. J Hydraul Eng 123:617-623

[34] Balmforth NJ, Craster RV, Perona P, Rust AC, Sassi R (2007) Viscoplastic dam breaks and the Bostwick consistometer. J Non-Newton Fluid Mech 142:63-78

[35] Hogg AJ, Matson GP (2009) Slumps of viscoplastic fluids on slopes. J Non-Newton Fluid Mech 158:101-112

[36] Perona P (2005) Bostwick degree and rheological properties: an up-to-date viewpoint. Appl Rheol 15:218-229

[37] Hewitt DR, Balmforth NJ (2013) Thixotropic gravity currents. J Fluid Mech 727:56-82

[38] Tanner RI (2000) Engineering Rheology (2nd ed). Oxford University Press

[39] Myers TG (2005) Application of non-Newtonian models to thin film flow. Phys Rev E 72:066302

[40] Tanner RI, Walters K (1998) Rheology: an Historical Perspective. Elsevier

[41] Barnes HA, Hutton JF, Walters K (1989) An Introduction to Rheology. Elsevier

[42] Wilson SDR, Burgess SL (1998) The steady, spreading flow of a rivulet of mud. J NonNewton Fluid Mech 79:77-85

[43] Wilson SK, Duffy BR, Hunt R (2002) A slender rivulet of a power-law fluid driven by either gravity or a constant shear stress at the free surface. Q J Mech Appl Math 55:385408 
[44] Yatim YM, Wilson SK, Duffy BR (2010) Unsteady gravity-driven slender rivulets of a power-law fluid. J Non-Newton Fluid Mech 165:1423-1430 